\title{
EFECTO DEL ACEITE DE SACHA INCHI (Plukenetia volubilis) EN LA DIETA DE REPRODUCTORAS DE POLLOS DE ENGORDE SOBRE EL DESEMPEÑO PRODUCTIVO DE SU PROGENIE
}

\author{
Effect of Sacha Inchi Oil (Plukenetia volubilis) in the Diet of Broiler \\ Breeders on the Productive Performance of Their Progeny
}

Juan Lucas L. ${ }^{1,2}$, Eliana Icochea D. ${ }^{3,4}$, Ricardo Valdivia R. ${ }^{5 \dagger}$, Fernando Carcelén C. ${ }^{6}$, John Guzmán G. ${ }^{3}$

\section{RESUMEN}

\begin{abstract}
El objetivo del estudio fue evaluar el efecto de la adición del aceite de sacha inchi (Plukenetia volubilis) en la dieta de reproductoras pesadas sobre los parámetros productivos de su progenie. Se utilizaron 720 pollos machos de la línea Cobb Vantress 500 procedentes de reproductoras alimentadas con una dieta conteniendo 5\% de aceite de Sacha inchi $(n=360)$ o con una dieta con grasa convencional $(n=360)$. Se usaron nueve repeticiones por grupo. Se evaluó el peso corporal, consumo de alimento, mortalidad, índice de conversión alimenticia (ICA), índice de eficiencia productiva europeo (IEP) y nivel de inmunidad humoral activa y pasiva. A los 42 días de edad, la inclusión de aceite de Sacha inchi en la dieta mostró un efecto positivo pero no significativo, en el comportamiento productivo de la progenie, e incrementó el nivel de inmunidad pasiva contra el virus de la enfermedad de Newcastle $(\mathrm{p}<0.05)$ y en forma no significativa contra los virus de Gumboro y bronquitis infecciosa.
\end{abstract}

Palabras clave: ácido graso polinsaturado, ácido graso linolénico, parámetros productivos, anticuerpos maternales

\section{Abstract}

The aim of the study was to evaluate the effect of Sacha inchi oil (Plukenetia volubilis) in the diet of broiler breeders on the productive performance of their progeny. The study included 720 male Cobb Vantres 500 chicks where half of them originated from breeders fed with a diet containing 5\% Sacha inchi oil and the other half fed with a diet containing conventional fat. Nine replicates per group were used in the study. Body weight, food intake, mortality, feed conversion index (FCI), European productive efficiency index (PEI),

\footnotetext{
${ }^{1}$ Laboratorio de Salud Pública y Salud Ambiental, ${ }^{3}$ Laboratorio de Patología Aviar, ${ }^{5}$ Profesor Emérito,

${ }^{6}$ Laboratorio de Bioquímica, Nutrición y Alimentación Animal, Facultad de Medicina Veterinaria, Universidad Nacional Mayor de San Marcos, Lima

${ }^{2}$ E-mail: mv.jrlucas@hotmail.com

${ }^{4}$ E-mail: eliana.icochea@gmail.com
} 
and level of active and passive humoral immunity were evaluated. At 42 days of age, the inclusion of Sacha inchi oil in the diet showed a positive but not significant effect on productive performance, but a significant increase in the level of maternal antibodies against Newcastle disease $(\mathrm{p}<0.05)$ and a slight increase of maternal antibodies against Gumboro and infectious bronchitis viruses.

Keywords: polyunsaturated fatty acid, linolenic fatty acid, poultry production parameters, maternal antibodies

\section{INTRODUCCIÓN}

El desempeño productivo en el pollo de engorde depende, en parte, de la calidad nutritiva del huevo incubable. Los ácidos grasos de la yema representan más del $90 \%$ del total de los requerimientos energéticos para el desarrollo, producción de energía y síntesis estructural de membranas de los embriones (Hargis et al., 1992; Latour et al., 1998; Speake et al., 1998; Hall et al., 2007), por lo que existe un gran interés en el rol de los ácidos grasos maternales en la modulación de la salud de la progenie.

El tipo de ácido graso (saturado o insaturado) determina las propiedades y la capacidad de intercambios fisiológicos de las membranas celulares (Mayes, 2001). Los ácidos grasos esenciales poliinsaturados (PUFA) modulan la expresión de genes que codifican las proteínas envueltas en la inflamación, metabolismo lipídico y la utilización de energía, así como en procesos relacionados a la inmunidad innata y adquirida (Deckelbaum et al., 2006).

La estructura química de los PUFA depende de la familia de origen. La familia n-6 deriva del ácido graso linoleico (LA) o ácido graso omega 6 , y la familia n-3 deriva del ácido graso alfa linolénico (ALA) o ácido graso omega 3 (Van Elswyk, 1997; Cherian y Sim, 2001; Leaf et al., 2003; Carrero et al., 2005). El embrión evita usar los PUFA para la beta oxidación, pues los requiere para la síntesis de los lípidos de membrana y eicosanoides (Neuringer et al., 1988; Speake et al., 1998; Cherian, 2008).

En el cuerpo de los animales, el LA es elongado y desaturado para formar el ácido araquidónico (AA), C20:4 n-6. El ALA emplea y compite por las mismas rutas metabólicas y enzimas para formar el ácido eicosapentaenoico (EPA), C20:5 n-3 (Cherian y Sim, 2001; Leaf et al., 2003; Knoch et al., 2009). El EPA es elongado a ácido docosapentaenoico (DPA), C22:5 n-3, para producir finalmente el ácido graso más largo y más insaturado normalmente encontrado en las dietas, el docosahexaenoico (DHA), C22:6 n-3. El EPA y el AA se incorporan a las membranas de las células, pudiendo ser liberados por la fosfolipasa $\mathrm{A} 2$, siendo luego sustratos para la ciclooxigenasa y lipooxigenasa, produciendo unos mediadores celulares llamados eicosanoides, los cuales intervienen en numerosos procesos fisiológicos como la coagulación de la sangre y la respuesta inmunológica. Los eicosanoides comprenden las prostaglandinas, tromboxanos, leucotrienos, lipoxinas y resolvinas (Cherian y Sim, 2001; Bannemberg et al., 2007; Knoch et al., 2009).

Los eicosanoides n-6, derivados del AA, ejercen propiedades proinflamatorias en su mayoría, además de liberar radicales libres que causan daños importantes a las membranas celulares. El EPA es la fuente de los eicosanoides n-3, los cuales en su mayoría tienen actividad antiinflamatoria, remueven las células inflamatorias y restablecen la integridad tisular (Dommels et al., 2002; Mills et 
al., 2005; Schmitz y Ecker, 2008; Serhan y Chiang, 2008).

El ALA se incorpora en las membranas celulares mejorando su flexibilidad y con componentes que producen menor actividad inflamatoria; lo que resulta en la prevención de problemas metabólicos e inflamatorios, que son afecciones comunes en aves de rápido crecimiento. También tiene un efecto positivo sobre los niveles de anticuerpos maternales, lo que podría aumentar la productividad (Ajuyah et al., 2003; Wang et al., 2004; Cherian, 2007). Se ha demostrado que el ALA estimula la unión de la IgY con su receptor en la membrana del saco vitelino, incrementando la transferencia maternal embrionaria de anticuerpos maternales (Wang et al., 2000; Wang, 2001).

Se ha experimentado con diversas fuentes locales de ácido graso linolénico, incluyendo fuentes vegetales y animales. En la Amazonía peruana existe el Sacha inchi (Plukenetia volubilis), especie vegetal cuya semilla es rica en ácidos grasos linolénicos y en vitamina A y E (Pascual y Mejía, 2000), y cuya producción podría ser una alternativa importante al cultivo de coca. A pesar de sus bondades, se dispone de pocos estudios como fuente de PUFA (Hamaker et al., 1992; Guillén et al., 2003; Krivankova et al., 2007). Ensayos previos muestran que dietas con 5\% de aceite de sacha inchi en gallinas en producción presentaron el mejor contenido de omega 3 en los huevos (R. Valdivia, datos no publicados). Asimismo, ha sido demostrado que estas dietas deben ser ofrecidas por cuatro semanas previas a la recolección de los huevos fértiles a fin de obtener contenidos estables de ALA, EPA y DHA en la yema (Hargis et al., 1991; Van Elswyk, 1997).

El objetivo del presente estudio fue determinar si el aceite de Sacha inchi adicionado en la dieta de reproductoras de pollos de engorde mejora los parámetros productivos de su progenie.

\section{Materiales y Métodos}

El estudio se realizó en el galpón experimental de la Facultad de Medicina Veterinaria, Universidad Nacional Mayor de San Marcos, Lima. Se utilizaron 720 pollos de carne de la línea Cobb Vantress 500, de un día de edad, machos, divididos en dos tratamientos de acuerdo a la alimentación que recibieron sus progenitoras. Estas fueron parte de un mismo lote de reproductoras, alimentadas con una misma dieta pero con diferentes fuentes de grasa: T0 con grasa convencional y T1 con 5\% de aceite sacha inchi durante 4 semanas previas a la recolección de los huevos fértiles.

Se calculó el tamaño muestral mínimo mediante la fórmula de diferencia entre medias, dando como resultado 34 aves por tratamiento. Debido a esto, el estudio fue diseñado en base a nueve repeticiones de 40 pollitos por tratamiento. Las aves recibieron el mismo alimento, manejo y programa de vacunación, y fueron criadas sobre piso de cemento con cama de viruta de madera, a una densidad de 10 aves $/ \mathrm{m}^{2}$.

Se determinó la tasa de mortalidad en base a las pérdidas diarias y semanales de cada grupo hasta los 42 días de edad. Asimismo, se calculó el índice de conversión alimenticia (ICA), el Índice de eficiencia productiva europeo (IEP) y la uniformidad.

El ICA se define como la cantidad de alimento requerido para producir un kilogramo de peso vivo de pollo. Para esto, las aves fueron pesadas en forma individual y semanalmente durante las seis semanas del estudio. Además, se pesó el alimento ofrecido y el residual para calcular el alimento consumido en forma semanal. Se utilizó una balanza digital de $10 \mathrm{~kg}$ de capacidad y $0.1 \mathrm{~g}$ de precisión.

El IEP europeo evalúa el rendimiento productivo integral de una parvada de pollos 
Cuadro 1. Parámetros productivos a los 42 días de edad de pollos de engorde procedentes de un lote de reproductoras alimentadas con una fuente de grasa convencional o con aceite de sacha inchi (Plukenetia volubilis)

\begin{tabular}{lcc}
\hline \multirow{2}{*}{ Parámetros } & \multicolumn{2}{c}{ Dieta con } \\
\cline { 2 - 3 } & Grasa convencional & Aceite de sacha inchi \\
\hline Peso corporal (kg) & 2.651 & 2.673 \\
Ganancia de peso (kg) & 2.612 & 2.634 \\
Consumo de alimento (kg) & 4.575 & 4.574 \\
Mortalidad (\%) & 3.1 & 3.3 \\
Viabilidad (\%) & 96.9 & 96.7 \\
Uniformidad (\%) & 78.2 & 79.6 \\
ICA $^{1}$ & 1.73 & 1.71 \\
IEP $^{2}$ & 348.3 & 354.7 \\
\hline
\end{tabular}

1 Índice de conversión alimenticia acumulado

2 Índice de eficiencia productivo europeo

de engorde. Para el cálculo se empleó la siguiente fórmula: IEP $=([$ Tasa de viabilidad $\mathrm{x}$ Ganancia de peso/día]/ICA) x 100. La uniformidad se calculó en base a la media $\pm 10 \%$ del peso promedio, y el número de aves dentro de este rango se expresó en porcentaje de la población.

Se comparó la respuesta inmune para el virus de la enfermedad de Newcatle (vENC), de la bronquitis infecciosa (vBI) y de Gumboro, tanto pasiva al primer día de edad como activa al final de la crianza (42 días de edad), utilizando un kit comercial de ELISA (Laboratorios IDEXX) para la medición de anticuerpos.

$\mathrm{Al}$ azar, se colectaron 15 muestras de sangre por tratamiento en el día 1 para determinar el estado inmune de las aves y 20 muestras de sangre por tratamiento en el día 42 para evaluar la respuesta al programa de vacunación aplicado (Día 1: vacunas contra la enfermedad de Marek vía subcutánea, y contra Newcastle y Bronquitis Infecciosa vía aspersión; Día 10: vacuna contra la enfermedad de Gumboro [cepa 2512], vía ocular; y Día 18: vacuna contra Newcastle [cepa entérica VG/VAV], vía ocular).

El peso vivo promedio se analizó con la prueba $t$ de Student para muestras independientes. Los parámetros productivos se calcularon como promedios por corral. Asimismo, se determinó, mediante la prueba de Kolmogorov-Smirnov para una muestra, que todos los valores seguían una distribución normal. En base a esta normalidad, los datos se analizaron con la prueba de T de Student para muestras independientes. Se empleó el paquete estadístico SPSS.

\section{Resultados}

No se encontró diferencias estadísticas entre promedios en los parámetros productivos al día 42 de edad entre los dos grupos (Cuadro 1). Los promedios de títulos de anticuerpos en el día 1 y 42 se muestran en el Cuadro 2, donde solo se encontró diferencia en los niveles de inmunidad pasiva para ENC al día 1. 
Cuadro 2. Títulos de anticuerpos maternales y de respuesta inmune activa en pollos de engorde procedentes de un lote de reproductoras alimentadas con fuente de grasa conven cional (T0) o con aceite sacha inchi (Plukenetia volubilis) (T1)

\begin{tabular}{lcccc}
\hline & \multicolumn{3}{c}{ Promedio aritmético del título de anticuerpos } \\
\cline { 2 - 5 } Agente viral & \multicolumn{3}{c}{ Día 1 } & \multicolumn{3}{c}{ Día 42 } \\
\cline { 2 - 5 } & T0 & T1 & T0 & T1 \\
\hline Enfermedad de Newcastle & $7692.5^{\mathrm{a}}$ & $9783.3^{\mathrm{b}}$ & 238.2 & 236.1 \\
Gumboro & 4006.4 & 4623.6 & 2170.5 & 2650.1 \\
Bronquitis infecciosa & 3906.8 & 5099.2 & 237.7 & 415 \\
\hline a,b & \multirow{2}{*}{ Superíndices diferentes dentro de filas y días de medición muestran diferencia estadística $(\mathrm{p}<0.05)$}
\end{tabular}

\section{Discusión}

En una dieta típica comercial para reproductoras, como la utilizada en el grupo control del presente experimento, el contenido de PUFA n-6 y de grasas saturadas es abundante, lo que implica membranas poco flexibles en el desarrollo embrionario, que producen eicosanoides $n-6$, los cuales liberan radicales libres que causan daño a las membranas celulares durante el desarrollo embrionario y post eclosión (Dommels et al., 2002; Leaf et al., 2003; Cherian, 2007, 2008).

Estudios previos sobre el uso de los PUFA estuvieron limitados a determinar el efecto de los ácidos grasos maternales sobre la composición de los ácidos grasos de los tejidos de la progenie. Ajuyah et al. (2003) y Cherian (2007) demostraron que la dieta materna es de mayor importancia para conseguir niveles elevados de PUFA n-3 en la progenie. La dieta T1con aceite de sacha inchi empleada en el presente estudio debía resultar en un pollito de mejor constitución y calidad, lo que se reflejaría en los parámetros productivos al final de campaña. Sin embargo, si bien se observaron algunas tendencias de mejora productiva, no se encontraron diferencias estadísticas; no obstante, es posible, que de haberse continuado con la misma dieta en la progenie, como ocurrió en los experimentos de Ajuyah et al. (2003) y Cherian (2007) se hubiesen consolidado las diferencias entre grupos.

Los niveles de anticuerpos contra los virus de BI, Gumboro y ENC fueron ligeramente superiores en el grupo cuyas progenitoras recibieron la dieta con sacha inchi; aunque estos resultados solo fueron estadísticamente significativos para ENC (9783.3 y 7692.5) al primer día de edad $(\mathrm{p}<0.05)$, tal y como fuera demostrado por Wang (2001) y Wang et al. (2004) con dietas con alto contenido de ALA. Estos autores señalaron que la concentración total de IgY en el suero de las gallinas, en el embrión de 11 días, y en el pollito de un día de edad está directamente interrelacionada, indicando que la proporción de LA/ALA en la dieta materna podría modificar la concentración total de IgY en la yema de huevo y en el pollo bebé.

Los resultados observados en el presente estudio, en relación a los efectos de los PUFA n-3 de la dieta materna sobre la respuesta inmune de la progenie, indican que pueden ser usados en la alimentación de las reproductoras para incrementar la salud y productividad de la progenie. Sin embargo, sería recomendable realizar más experimentos para evaluar el efecto inmunomodulador, 
que incluyan desafíos de campo como indicador de protección inmune; asimismo, ensayos complementarios con aceite de sacha inchi en la dieta maternal y de la progenie.

\section{Conclusiones}

La inclusión de aceite de sacha inchi en la dieta de reproductoras pesadas no tuvo un efecto significativo en el comportamiento productivo de la progenie, pero incrementó el nivel de inmunidad pasiva contra el virus de la enfermedad de Newcastle al primer día de edad $(p<0.05)$.

\section{Literatura Citada}

1. Ajuyah O, Cherian G, Wang $Y$, Sunwoo H, Sim JS. 2003. Maternal dietary fatty acids modulate the longchain n-3 PUFA status of chick cardiac tissue. Lipids 38: 1257-1261.

2. Bannemberg G, Arita M, Serhan CN. 2007. Endogenous receptor agonists: resolving inflammation. Sci World J 7: 1444-1462.

3. Carrero JJ, Martín-Bautista E, Baró L, Fonollá J, Jimenez J, Boza JJ, Lopez-Huertas E. 2005. Efectos cardiovasculares de los ácidos omega-3 y alternativas para incrementar su ingesta. Nutr Hosp 20(1): 63-69.

4. Cherian G, Sim JS. 2001. Maternal dietary $\alpha$-linolenic acid (18:3 n-3) alters n-3 polyunsaturated fatty acid metabolism and liver enzyme activity in hatched chicks. Poultry Sci 80: 901-905.

5. Cherian G. 2007. Metabolic and cardiovascular diseases in poultry: Role of dietary lipids. Poultry Sci 86: 10121016.

6. Cherian G. 2008. Egg quality and yolk polyunsaturated fatty acid status in relation to broiler breeder hen age and dietary n-3 oils. Poultry Sci 87: 11311137.
7. Deckelbaum RJ, Worgall TS, Seo T. 2006. N-3 fatty acids and gene expression. Am J Clin Nutr 83: 1520S1525S.

8. Dommels YEM, Alink GM, Van Bladeren PJ, Van Ommen B. 2002. Dietary n-6 and n-3 polyunsaturated fatty acids and colorectal carcinogenesis: results from cultured colon cells, animal models and human studies. Environ Toxicol Phar 12: 233-244.

9. Guillén MD, Ruiz A, Cabo N, Chirinos R, Pascual G. 2003. Characterization of sacha inchi (Plukenetia volubilis L.) oil by FTIR spectroscopy and 1H NMR. Comparison with linseed oil. J Am Oil Chem Soc 80: 755-762.

10. Hall JA, Jha S, Skinner MM, Cherian G. 2007. Maternal dietary n-3 fatty acids alter immune cell fatty acid composition and leukotriene production in growing chicks. Prostag Leukotr Ess 76: 19-28.

11. Hamaker BR, Valles C, Gilman $R$, Hardmeier RM, Clark D, Garcia HH, Gonzales AE, Kohlstad I, Castro M, Valdivia R, Rodriguez T, Lescano M. 1992. Amino acid and fatty acid profile of the Inca peanut (Plukenetia volubilis). Cereal Chem 69: 461-463.

12. Hargis PS, Van Elswyk ME, Hargis BM. 1991. Dietary manipulation of yolk lipid with menhaden oil. Poultry Sci 70: 874-883.

13. Hargis PS, Van Elswyk ME, Coco CM. 1992. Effects of graded levels of dietary menhaden oil on amega-3 fatty acid incorporation into yolk lipids. Poultry Sci 71 (Suppl 1): 154.

14. Krivankova B, Polesny Z, Lojka B, Lojkova J, Banout J, Preininger D. 2007. Sacha inchi (Plukenetia volubilis, Euphorbiaceae): a promising oilseed crop from Peruvian Amazon. Conference on International Agricultural Research and Development. Germany: University of Kassel.

15. Knoch B, Barnett MPG, Roy NC, McNabb WC. 2009. Study of the effects of dietary polyunsaturated fatty 
acids: Molecular mechanisms involved in intestinal inflammation. Grasas Aceites 60(1): 8-21.

16. Latour MA, Peebles ED, Doyle SM, Pansky T, Smith TW, Boyle CR. 1998. Broiler breeder age and dietary fat influence the yolk fatty acid profile of fresh eggs and newly hatched chicks. Poultry Sci 77: 47-53.

17. Leaf A, Kang JX, Xiao YF, Billman GE. 2003. Clinical prevention of sudden cardiac death by n-3 polyunsaturated fatty acids and mechanism of prevention of arrhythmias by n-3 fish oils. Circulation 107: 2646-2652.

18. Mayes P. 2001. Metabolismo de los ácidos grasos insaturados y de eicosanoides. En: Murray R, Granner D, Mayes P, Rodwell (eds). Bioquímica de Harper. México: Manual Moderno. p 289-298.

19. Mills SC, Windsor AC, Knight SC. 2005. The potential interactions between polyunsaturated fatty acids and colonic inflammatory processes. Clin Exp Immunol 142: 216-228.

20. Neuringer M, Anderson GJ, Connor WE. 1988. The essentiality of n-3 fatty acids for the development and function of the retina and brain. Ann Rev Nutr 8: 517-541.

21. Pascual G, Mejía M. 2000. Extracción y caracterización del aceite de sacha inchi (Plukenetia vulubilis). Anales Científicos UNALM 42: 144-158.

22. Schmitz G, Ecker J. 2008. The opposing effects of n-3 and n-6 fatty acids. Prog Lipid Res 47: 147-155.

23. Serhan CN, Chiang N. 2008. Endogenous pro-resolving and antiinflammatory lipid mediators: a new pharmacologic genus. Brit J Pharmacol 153: S200-S215.

24. Speake BK, Noble RC, Murray AMB. 1998. The utilization of yolk lipids by the chick embryo. Poultry Sci 54: 319-334.

25. Van Elswyk, M. 1997. Nutricional and physiological effects of flax seed in diets for laying fowl. Poultry Sci 53: 253-264.

26. Wang YW, Sunwoo HH, Cherian G, Sim JS. 2004. Maternal dietary ratio of linoleic acid to $\alpha$-linolenic acid affects the passive immunity of hatching chicks. Poultry Sci 83: 2039-2043.

27. Wang YW. 2001. Omega-3 polyunsaturated fatty acids and chicken immunity. PhD Thesis. Canada: Alberta University. 222 p.

28. Wang YW, Cherian G Sunwoo HH, Sim JS. 2000. Dietary polyunsaturated fatty acids significantly affect laying hen lymphocyte proliferation and immunoglobulin $\mathrm{G}$ concentration in serum and egg yolk. Can J Anim Sci 80: 597-604. 